$\S=-1$

\title{
IoT System for Detecting Input/Output Patient \& Staff via RFID Technology
}

\author{
Sohaib Chengaou ${ }^{1 *}$, Khalid El Yassini $^{2}$, Kenza Oufaska $^{3}$, Tarik Zouadi ${ }^{4}$ \\ ${ }^{I}$ IA Laboratory, Faculty of Sciences Meknès, Moulay Ismail University, Morocco \\ ${ }^{2}$ IA Laboratory, Faculty of Sciences Meknès, Moulay Ismail University, Morocco \\ ${ }^{3}$ TICLab Laboratory, International University of Rabat \\ ${ }^{4}$ BEAR Lab, Rabat Business School, International University of Rabat \\ *Corresponding author E-mail:Sohaib.Chengaou@gmail.com
}

\begin{abstract}
The Internet of Things (IoT) is a concept based on several technologies by connecting them to create a network of networks and a system of systems. It is composed of several and different objects such as Smartphone, Smart Watch, Smart refrigerator, Smart tv, etc. And it can guarantee quick access to information, this has had an enormous influence on the pace of contemporary daily life. It is possible to use IoT in critical areas as health for better management of hospital structures or to improve working conditions and staff performance in the hospital domain. This work proposes a solution allowing an improvement in term of management of inputs and outputs of the employees and patients and thus a better management within a hospital.
\end{abstract}

Keywords: Internet of Things; RFID; Ubiquitous Computing; Health

\section{Introduction}

The Internet of thing is a new paradigm that uses daily objects such as Smartphone, Smart Watch, refrigerator, Smart TV, etc. by connecting them via network. Those objects can exchange their data without human interaction. This technology (IoT) represents a new generation of Web 2.0, and it is based on using different hardware, systems and technologies. The components heterogeneity induce another concept which is the "interoperability", that mean the ability of system to work with or use the parts or equipment of another system. It can also be called "network of networks" or "System of Systems". Before existing the internet of Things many technologies used to identify and transmit data, they have paved the path for a new concept who will be the dominant technology in the world. In this regard, we cite bar code, RFID technology, sensor networks, etc. [1].

Barcode: it is a graphic codification of information, i.e. a text, a number, or a character, etc. It allows the identification of an article or product.

RFID: it corresponds to an identification technology, which is appeared to compensate for the shortcomings of the bar code. The device is composed of RFID tag that represents the identifier of desired object to follow it, and a reader that has role in reading data belonging to the tags. RFID technology is applicable in different areas such as health, demotics, logistics and transport, etc. Network of sensors (WSN): It is composed of a set of sensors deployed either in a random manner or by specifying the locations of the nodes, each node is considered as a router which routes data from a source to a destination. WSN is applicable in different areas such as the medical follow-up, medical surveillance, environmental monitoring, intelligent buildings and the mapping of the biodiversity. WSN is able to auto-configure and adapt despite the changes of the topologies, which offers a more for this technology. All indispensable domains can benefit from the IoT concept and integrate it to improve the quality of work and the offered services. Among the fertile domains that requires the integration of the Internet of Things, there is the agricultural domain, the industrial domain, logistics and transport domain and health domain. It should be noted that all these areas need more evolution to achieve the desired objectives. In this regard, Venot $\&$ al. [4] identify and describe a multiplicity of solutions dedicated to health domain. This work proposes a solution to two major problems related to health management domain in Morocco. The first problem concerns the escape of patients from hospitals (or babies kidnapping, etc) without regularizing administrative situations or without the agreement of corresponding doctors, etc. While the second problem is related to staff management, resolution of those problems with a direct impact on the quality of staff's work and on administration of hospital.

The proposed solution is a system called 'IoT-SDES2P' aimed to detect the input / output of staff and patients using RFID technology while providing mechanisms for the presence validation and information management of staff and patients.

\section{Iot-SDES2P System}

The proposed solution is a system for identifying and detecting the inputs and outputs of patients and employees, it is based on RFID technology. This technology identifies staff and patients in a fast way and with a safety of their identities, it is composed of a tag RFID and a RFID reader. The tag contains a unique identifier that has hexadecimal form. The recovery of this identifier is done by the RFID reader which reads the tag-RFID [2] in order to transmit the code or to use it to make other treatments. 


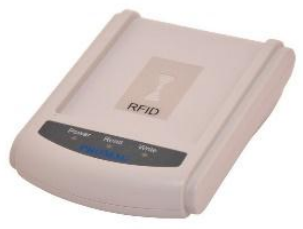

Fig. 1: RFID Reader

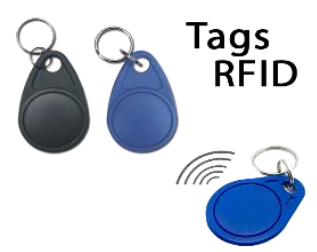

Fig. 1: Tag RFID
The system develops two main scenarios that are as follows:

\section{A staff scenario}

It is possible to divide this scenario into two scenarios:

- The first scenario starts at the arrival of a new employee (doctor, nurse, maid, ...), the administrator will add his information to the database, then he will integrate the identifier of a tag-RFID with the information of the new employee, so that it will be referenced by this identifier and he will assign a profile.

- As regards the second scenario, it begins when an employee will put his tag-RFID in front of the reader to validate his presence, the reader will recover the tag code then it will be transmute in to Java application which treats it. i.e. it ensures What does the code refers to, either a patient or an employee, if the code refers to an employee application will validate his presence with the date and time of posting.

A patient scenario

It is possible to divide this scenario into two scenarios:

- As soon as a patient arrives to the hospital, the administrative process begins by adding his personal information to the system. Among the information entered by the hospital reception officer, the tag code that will allow the identification of this patient, then the agent give him a mobile bracelet that contains the tag to identify him when he wants to get out of the hospital.

- When a patient wants to leave the hospital, the RFID reader detects the tag, then it lights a lamp and it triggers a sound as indication to know what the patient is doing. i.e. patient wants to go out or escape without resolving its administrative situation. After reading the tag code, it will be transmitted to a Java application that searches in the database for information referenced by it, then displays it on a window, this -information shows the medical situation of the patient and is it checks if he has an exit agreement from his doctor and according to this information the agent authorizes the exit of the patient or he refuses it.

The system is divided into two parts, the first part for detecting tag code, this part has a program which allows to read the code and transmits it via a serial port to a Java application installed in hospital reception officer station, while the second part is processing the tag code that will allow a receptionist to manage the patient information.

To see the benefits of such a system, we take two hospitals, the first uses an old system of management of patient and staff entries and exits, and the second hospital uses a new management system based on new technologies such as the IoT-SDES2P system to achieve better management. Figure 3 represents an explanation of second scenarios - patient scenario - of the proposed system, it shows the elements constituting the scenario as well as the components of the system.

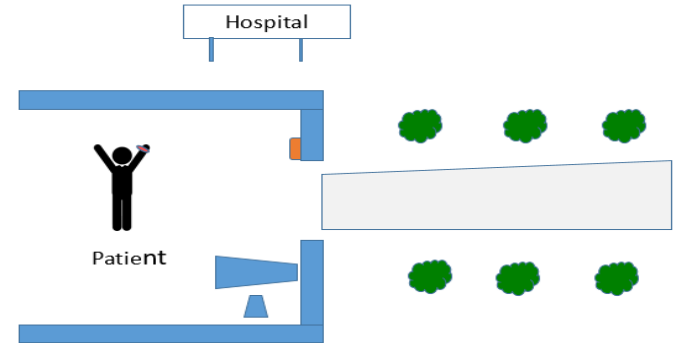

Fig. 3: explanation of second scenario of the proposed system

Hospital that uses SDES2P will be able to identify the outputs of patients quickly and in real-time with a security of their identities, while the other hospital will always be the problem of run away of patients.

\subsection{System demonstration}

Fig.3 represents an authentication window which allows an employee who has an account to authenticate. The employee can have an account if he have one of these profiles:

- Hospital reception officer

- Administrator

- Owner of a private hospital

- Doctor

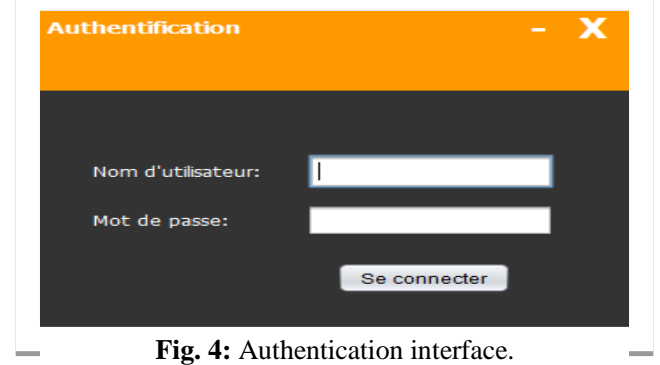

Fig. 4 is an authentication interface which allows different users to authenticate in order to access to its work interfaces as figures 5, 6 show.

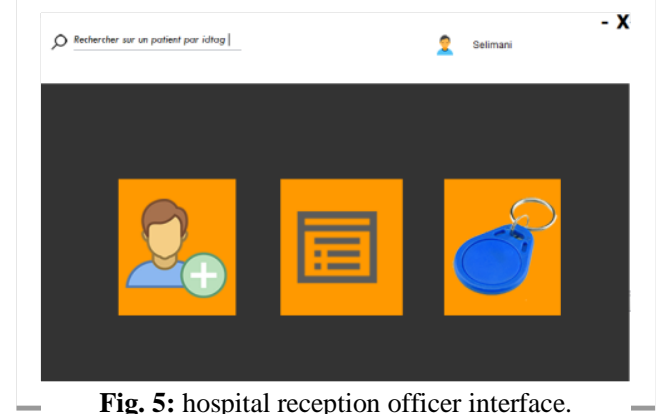

Fig.5 shows the receptionist interface, he can add patient informations, consult patients list and modify their information, and he can also know the tag code for using the tag RFID. 


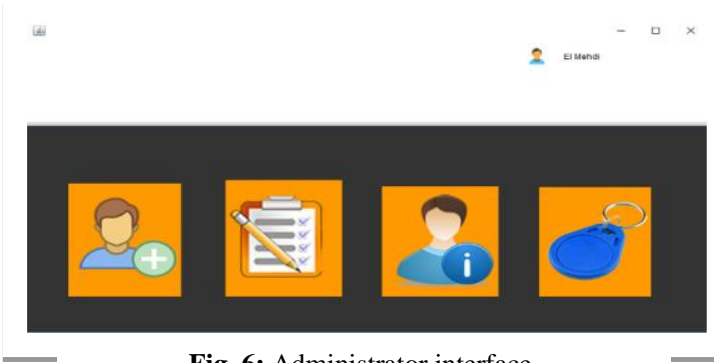

Fig. 6: Administrator interface.

Similar to FIG. 5, FIG. 6 corresponds to administrator home interface who can add and see employees lists for any modification or deletion.

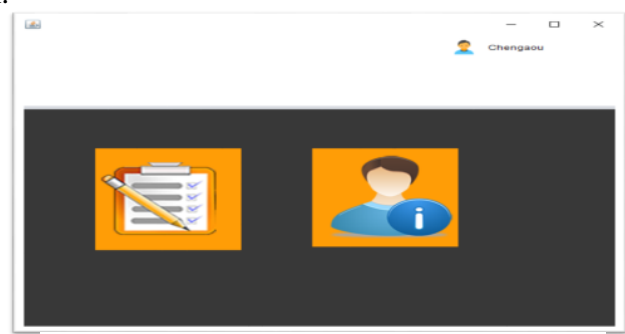

Fig. 7: Doctor interface.

There is another interface [Fig. 7], it is a doctor interface that allows doctor to see his patients list and he can validate the patient output from hospital.

\section{Discussion and Criticism}

No one can deny that health represents crucial area in the countries of the third world and also to the Morocco. Moroccan health sector has several challenges that push young researchers to propose a few solutions to improve work conditions to the staff and the quality of the services offered to patients.

The proposed system has positive points and points that can be discussed in order to strengthen and evolve it, it is difficult to deal all of them, but at least the points that are essential and have a budget and quality services impact.

The positives of the proposed system, include the following:

- The easiness of identification of employees and patients;

- Decrease or elimination of absenteeism or employees delay;

- Improving employees performance

- Decrease in the phenomenon of patients escape not justified

- Etc.

The positives of the proposed system include the following:

- The easiness of identification of employees and patients;

- Decrease or elimination of absenteeism or employees delay;

- Improving employees performance;

- Decrease in the phenomenon of patients escape not justified

\section{Conclusion}

It's obvious that the world is becoming more and more connected, and a technology able to keep and evolve this concept corresponds to Internet of Things since it allows interconnecting the everyday objects to accelerate the process of accessing to information. It should be adopted and used in critical areas such as health to evolve working conditions and staff performance to save lives. The proposed system is a practical solution to solving some health field problems that still requires ideas to improve this critical area.

\section{Acknowledgement}

The authors would like to thank all committee of the International Conference of technology, Innovation and Information systems (CITISI/2), for their organization and supports.

\section{References}

[1] Andrew Whitmore, Anurag Agarwal and Li Da Xu, « The Internet of Things: A survey of topics and trends », Information Systems Frontiers, Vol. 17 (2), Kluwer Academic Publishers, pp. 261-274, 2015.

[2] Ammar Rayes and Samer Salam, « Internet of Things From hype to Reality - The Road to Digitization », Springer International Publishing, pp. 67-70, 2017

[3] FTC Staff Report, « Internet of Things Privacy \& Security in a Connected World »

[4] Alain Venot, Anita Burgun, et Catherine Quantin, «Informatique médicale, e-Santé Fondements et applications », Springer Verlag France, 2013 